\title{
Ciudadanía, educación y la realidad brasileña: Puntos de debate y encaminamientos*
}

\author{
Citizenship, education and brazilian reality: Discussion points and aimings \\ Cidadania, educação e realidade brasileira: Pontos de debate e encaminhamentos
}

\author{
Lilian Tatiane Candia de Oliveira, ${ }^{\mathrm{a}}$ Paulo Gomes Lima ${ }^{\mathrm{b}}$
}

${ }^{\mathrm{a}}$ Universidad Federal da Grande Dourados [UFGD], Facultad de Educación, Dourados/MS-Brasil. Telf.: 55(67) 9171-1817. Correo electrónico: lilian.candia@ hotmail.com

${ }^{\mathrm{b}}$ Universidad Federal da Grande Dourados [UFGD], Facultad de Educación, Dourados/MS-Brasil. Telf.: 55(15) 91028974. Correo electrónico: paulolima@ufgd.edu.br

\begin{abstract}
RESUMEN
Este estudio discute la ciudadanía desde una perspectiva recurrente a través de un análisis crítico y reflexivo en cuatro secciones. En la primera analiza los antecedentes históricos de la ciudadanía en las condiciones y valores que atendieron al hombre y su comprensión de la realidad. En la segunda sección, distinguimos los elementos estructurales de la consolidación del término ciudadanía y las contradicciones de su reflexión. A continuación, debatimos la ciudadanía en la actualidad y, finalmente, recurrimos a los desafíos y encaminamientos de la ciudadanía para la educación en Brasil. El estudio muestra que la perspectiva democrática mejora los encaminamientos de las políticas educativas y que su significado en la construcción de las personas que viven y reclaman el espacio social, como conquista universalizada y humanizada, aun sigue siendo una realidad en construcción.
\end{abstract}

Palabras clave: ciudadanía, sentidos, significados, políticas educativas.

\begin{abstract}
This paper discusses the appellant citizenship perspective through a critical and reflective analysis of four sections. The first analyze the historical background of citizens under the conditions and values who treated them and their understanding of reality. In the second section, Citizenship and contradictions: meanings and significance, we distinguish the structural elements of the consolidation of the term citizenship and the contradictions of its reflection. Then in "educational policy and citizenship" current citizenship is debated; and finally in the fourth section we turn to the challenges and routing of citizenship education in Brazil. The study shows that the democratic perspective improves the development of educational policies in the construction of meaning where people live and claim social space conquest humanized universalized and still remains a reality in construction.
\end{abstract}

Key words: citizenship, senses, meanings, educational policies.

\section{RESUMO}

Discute-se a cidadania a partir de uma perspectiva recorrente por meio de uma análise crítica e reflexiva em quatro seções. A primeira analisa o contexto histórico de cidadania nas condições e valores que atenderam ao homem e à sua compreensão da realidade. Na segunda, Cidadania e contradições: sentidos e significados, distingue-se os elementos estruturais da consolidação do término da cidadania e as contradições de sua reflexão. Na sequência, em "Política educacional e cidadania", debate-se a cidadania na atualidade e, finalmente, na quarta seção, recorre-se aos desafios e encaminhamentos da cidadania para a educação no Brasil. Mostra-se que a perspectiva democrática melhora os encaminhamentos das políticas educativas para efetivação de seu significado na construção de pessoas que vivam e reclamem espaço social como conquista universalizada e humanizada, embora sendo esta uma realidade em construção.

Palavras chave: cidadania, sentidos, significados, políticas educacionais.

\footnotetext{
* Trabajo financiado por la Coordinación de Perfeccionamiento de Personal de Nivel Superior (CAPES-Brasil).
} 


\section{INTRODUCCIÓN}

La propiedad del caleidoscopio es presentar varias fases del objeto y posibles combinaciones, por lo tanto, las imágenes que se forman a partir de una perspectiva presentan particularidades al observador. En este sentido, aunque sin la intención de realizar o de agotar todas las combinaciones, el propósito de este artículo es, apropiándose de una mirada caleidoscópica delimitada, reunir pistas para problematizar algunos sentidos y significados sociales de la ciudadanía en la contemporaneidad, su contemplación en la políticas educativas y algunos encaminamientos referente a los desafíos que hay que afrontar con el fin de lograr el pleno sentido. Como supuesto metodológico entendemos que la crítica reflexiva en el marco del materialismo histórico (Lima, 2003) ${ }^{1}$ se presenta como el más adecuado para el tratamiento del objeto.

La perspectiva materialista histórica, entre sus dimensiones, recorre la dialéctica para construir su universo de problematizaciones y en ella, la unidad es lucha de los opuestos y uno de los puntos fundamentales para la comprensión del objeto. En este sentido, vamos a utilizar la perspectiva de la lucha de los opuestos en el análisis de la construcción social de la ciudadanía a partir de las contribuciones de Pinsky (2010), Guarinello (2010), Funari (2010), Hoornaert (2010) y Zeron (2010) como punto de partida para la contextualización de algunos antecedentes de la ciudadanía y de los marcos develadores de las condiciones materiales de existencia, expresado a veces por demostración éticoreligiosa o por la disposición estructural del modo de producción y sus consecuencias entre los sentidos y significados.

La constitución de los sentidos y significados de ciudadanía en la dimensión de las contradicciones del modo de producción entre el linealismo y la recurrencia (Marshall, 1967; Saes, 2012; Afonso y Ramos, 2007; Martins, 2000), son puntos centrales del debate sobre el contexto de su aplicación en constante transformación; ya sea por políticas públicas en su conjunto para garantizar las condiciones adecuadas para su realización, tanto en las políticas sectoriales tales como las educativas, que entre otras cosas a lo largo de los años ha hecho hincapié en "la formación de los actores sociales para ejercer la ciudadanía".

Tenemos en este caleidoscopio algunas cuestiones urgentes que requieren, por lo menos, un ensayo de respuesta, a saber: si se quiere formar al hombre para el ejercicio de la ciudadanía, ¿de qué ciudadanía se está hablando? ¿Qué tipo de hombre formar para qué tipo de sociedad? Pensando en la realidad brasileña, ¿cuáles encaminamientos y desafíos necesitan ser enfrentados para no abordar solamente la problemática, sino por la seguridad de los ciudadanos en el pleno sentido? Todas estas cuestiones son objeto de este trabajo que entiende que la dimensión de la democratización y humanización no puede estar desvinculada de la horizontalidad de la justicia y la legalidad en sentido universal y material de lo que se entiende por ciudadanía.

A efectos de la exposición del trabajo organizamos cuatro secciones para reflejar la unidad del tema en cuestión. Durante la primera sección "Antecedentes históricos de la ciudadanía", vemos los primeros ensayos de una idea sobre ciudadanía desde la

\footnotetext{
1 De acuerdo con Lima (2001:26-27) la sociedad se estructura a partir de la forma como los hombres producen y organizan sus medios de vida. Marx y Engels (1996) explicitan: "tal como los individuos manifiestan su vida, así son ellos. Lo que son coincide, por lo tanto, con su producción, tanto con lo que producen, como con el modo cómo producen. Lo que los individuos son, por lo tanto, depende de las condiciones materiales de su producción".
} 
antigüedad hasta la contemporaneidad, seguido por el segundo apartado "Ciudadanía y contradicciones: sentidos y significados" en el que se presentan los argumentos sobre el sentido social lineal como propuesta de constitución de la ciudadanía y su defensa, teniendo en cuenta las diversas limitaciones que demuestran solicitudes conflictivas y significados no armoniosos en el proceso de los diferentes intereses sociales. Continuando con la exposición textual observamos en "La política educativa y la ciudadanía" los delineamientos y las reflexiones de la ciudadanía para la realidad brasileña, y finalmente, en la cuarta sección se analizan los "Desafíos y encaminamientos sobre la dimensión de la ciudadanía para la educación en Brasil".

\section{ANTECEDENTES HISTÓRICOS DE LA CIUDADANÍA}

La cuestión de la ciudadanía ha sido objeto de reflexión de numerosos estudiosos de las ciencias humanas y sociales que buscan problematizar esta cuestión en la perspectiva de profundizar el conocimiento respecto a esta categoría.

Aunque el concepto de ciudadanía se relaciona tradicionalmente con la revolución burguesa, con la realización de las libertades individuales, es necesario entender cómo la organización humana, en mayor o menor grado, ha orientado su manifestación en ese período y más allá de él para así comprender en qué aspectos las nuevas formas de ser, de vivir y de producir del hombre exigieron y permitieron el establecimiento de marcos regulatorios sobre su vida social.

Algunos autores sugieren la ocurrencia de las manifestaciones de la ciudadanía, incluso antes de su propia existencia, o en buenos términos franceses, "avant la letre", que se caracteriza como la prehistoria de la ciudadanía. Por lo tanto, tal proposición requiere una breve incursión histórica en su proceso evolutivo. Como sabemos, para recorrer un itinerario histórico, en la delimitación didáctica de los textos, algunas decisiones deben ser tomadas aun con el riesgo de no contemplar algunas manifestaciones también relevantes.

No tenemos la intención de desplegar la historia del arte de los antecedentes históricos de la ciudadanía, misión audaz para el propósito de este trabajo, impracticable incluso. Optamos por "dar una pincelada" a algunos contextos que podrían contribuir a la discusión de la ciudadanía en una perspectiva más general, incluso antes de su formación.

Jaime Pinsky (2010: 16), en un intento de identificar los fundamentos históricos más remotos de la ciudadanía, explica que estos se pueden encontrar en el legado del pueblo hebreo. No se encuentra en el cristianismo, y ni siquiera en el monoteísmo, sino en

[...] concepción de un dios que no se satisfacía en ayudar a los ejércitos, sino que exigía un comportamiento ético por parte de sus seguidores. Un dios poco preocupado en ser el objeto de la idolatría de la gente y el sacrificio de los animales en su holocausto, pero muy comprometidos con los problemas relacionados con la exclusión social, la pobreza, el hambre, la solidaridad (Pinsky, 2010: 16).

Pinsky (2010: 17) buscar detectar a lo largo de la historia una característica peculiar del pueblo hebreo, que culminó en una práctica que denomina "monoteísmo ético" y explica que "[...] el adoctrinamiento de los llamados profetas sociales establece los fundamentos del monoteísmo ético, [...] y es, probablemente, la primera expresión documentada y políticamente relevante (incluso por sus consecuencias históricas) de lo que podríamos llamar la prehistoria de la ciudadanía". 
Explicita que en el análisis de textos de diferentes autores judíos, de diferentes épocas y lugares, se pudo ver el orgullo en mostrar un comportamiento con una baja incidencia de borrachos, ladrones o asesinos. Uno puede detectar, también, una presión grupal mutua en la que prevalecía la amenaza de la marginación a los que se comportan incorrectamente. Así, "[...] desde las primeras letras a los seminarios rabínicos se enseñaba la necesidad de que cada judío de haga valer los valores de la justicia y de la fraternidad establecida por Dios y cumplidos por sus antepasados. En la ética práctica reside la diferencia fundamental entre judío y no judío "(Pinsky, 2010: 18).

No se puede dejar de notar, sin embargo, que la inducción de mantener esta ideología se llevó a cabo por convencimiento y la fe hebraica transmitida de padres a hijos como una manifestación de la voluntad trascendental, de ahí la aceptación de las condiciones comunales dadas y la legitimidad de los señores tribales sobre sus dominados. Aunque hubo toda una dimensión ética de valor religioso, el sentimiento de dominación ideológico era una situación que no se entendía como un problema, o se aceptaba o rechazaba. Puesto que la educación estaba dirigida a cumplir con el acuerdo "inculcado" por los sacerdotes desde el nacimiento del niño, no había ningún desafío a lo "normal" de establecido.

A partir de Simon Dubnow (un historiador importante), Pinsky relata que una nación puede pasar por tres etapas: lo tribal, lo político territorial y lo histórico cultural. Para Dubnow, solamente los judíos alcanzarán la última etapa, por lo tanto, extrapolaron las ligaciones territoriales y se mantuvieron como nación, aunque dispersos por el mundo. Sin embargo, la pregunta sigue siendo: ¿quién creó el monoteísmo ético? Y responde que se trata de una creación de los profetas, que se convirtieron en una conquista de toda la humanidad. Intrínseco en el individuo pervivió la doctrina de la primacía de la moral.

Pinsky (2010: 27) comenta los textos escritos por los profetas Isaías y Amós (en un periodo en que la monarquía ya estaba dividida entre Israel y Judá) para explicar la naturaleza política del pensamiento de esos profetas, que el autor denomina "revolucionario". Una vez que, con osadía (y bajo la dirección de Dios), se atrevieron a desafiar su tiempo, sus reyes y sus costumbres y a pregonar una práctica de vida que se preocupa por la justicia social, moral y económica en lugar de costumbres religiosas y prácticas egoístas, "renuncian al dios del templo, y cualquier templo y crean el dios de la ciudadanía" .

No se puede afirmar, sin embargo, que los ciudadanos con la vivencia moral o el establecimiento del estatuto moral judío se configuraron como un conjunto amplio y sin restricciones, toda vez que servía en el nivel de la mayor amplitud a los judíos libres y convertidos. La jerarquía de dominación se hacía por la fuerza militar y la extensibilidad de los territorios. La relación de servidumbre no era una cuestión de justicia social o algo así, puesto que no era un problema en su organización, dado el dominio completo de quien tenía la hegemonía teocéntrica y económica. Lo mismo se aplicaba a las mujeres, condicionadas como propiedades sus esposos, con una educación de acuerdo a su papel social, principio que se hacía valer para sirvientes e hijos.

El principio de extensión de las riquezas, los derechos y prerrogativas sociales a los "hombres de polis" tiene un fuerte componente de recurrencia en el hombre griego, sin embargo, el modus de reconocimiento en las polis se daba por nacimiento o por pertenecer a la aristocracia, pero no se puede negar que la forma de representación que comienza a dibujar su democracia, los espacios para la discusión y manifestación cultural, pues en Grecia: 
[...] está a la vista de los grandes pueblos de Oriente, el "progreso" fundamental, un nuevo "estadio" en todo lo que se relaciona con la vida de los hombres en la comunidad. Esto se basa en principios completamente nuevos. Por más elevadas que juzguemos las realizaciones artísticas, religiosas y políticas de los pueblos anteriores, la historia de lo que llamamos cultura con plena conciencia sólo comienza con los Griegos (Jaeger, 1995: 5).

En este sentido, Lima (2012) complementa que el sentido de la cultura, la educación y la pedagogía de la educación griega asume otro significado en relación con los pueblos anteriores, debido a que el factor de racionalidad sobre el mundo y sus limitaciones se produce a partir del hombre griego, ya sea por un saber contemplativo, ya sea por el conocimiento de la relaciones vividas, o por un énfasis en la articulación de ambos. En este sentido, la racionalidad (Logos) griega hace hincapié en un tipo diferente de educación y pedagogía centrada en la formación del hombre integral, sin embargo, uno no puede decir que todo el mundo tenía la misma condición horizontal de convertirse en un "ciudadano" de la polis, como se señaló anteriormente, incluso en la fase de una educación centrada en los valores aristocráticos.

Guarinello (2010), al discutir las contribuciones greco-romanas de la Antigüedad Clásica, explicita que comprender la ciudadanía requiere de conocimientos sobre un largo proceso histórico en el que el Imperio Romano es punto de paso obligatorio. Hace hincapié en que en este proceso se produjo la evolución de la idea de ciudadanía, que tiene su origen en la pertenencia a una comunidad agrícola pequeña (Ciudad-Estado) y que más tarde se convierte en una fuente de conflictos y reivindicaciones al entrechocar las diferentes concepciones de los derechos y deberes de los ciudadanos:

[...] La participación en el poder, la igualdad jurídica, y también la igualdad económica eran los términos en que proponían, en repetidas ocasiones, en estos conflictos, hasta que un Poder superior se estableció sobre el conjunto de las ciudades-estado y suprimió de la ciudadanía comunitaria progresivamente su capacidad de ser una fuente potencial de reivindicaciones (Guarinello, 2010: 45-46).

La cultura helenística presente en el Imperio Romano tendrá en cuenta la forma y disposición de las leyes, algunos beneficios sociales aplicadas a los ciudadanos romanos, sin embargo, dice Lima (2012), que el contenido cultural, movilizado por su forma de organización social, por el surgimiento sistematizado de la filosofía, hizo de la educación griega un hito en la historia de la educación y el pensamiento pedagógico romanos difundidos en la "civitas" del cual, incluso en la edad medieval, no se puede ignorar su importancia; sin embargo, sin dejar de delimitar cada segmento humano por su condición de nacimiento o jerarquía social.

El legado romano contribuyó en gran medida a la actual concepción de la ciudadanía, como lo demuestra Funari (2010: 76), que establece que la experiencia romana tuvo características muy similares a las nociones modernas de ciudadanía y participación popular. El autor señala que "[...] los padres fundadores de los Estados Unidos de América tomaron como modelo la constitución romana republicana, con la combinación del Senado y la Cámara (en lugar de las antiguas asambleas)" . Otro aporte fue el uso de votaciones secretas que Funari considera la piedra fundamental de la libertad ciudadana. También destaca la realización de Foros caracterizado como el mayor símbolo de la participación ciudadana. 
En la sociedad romana, más concretamente en las comunidades cristianas de los primeros siglos, se encuentra otro elemento impulsor de las bases de la ciudadanía. Sin embargo, esta afirmación no es evidente en los documentos que permiten la idea del cristianismo que articula la ciudadanía; hay que "[...] leer entre líneas, interpretar subentendiendo, investigar desde una nueva perspectiva, ya que los intelectuales de ese tiempo no mostraban interés por lo que sucedía en el interior del movimiento cristiano" (Hoonaert, 2010: 83).

Para este autor, el extraordinario desarrollo de las comunidades cristianas en tan poco tiempo no se debe (como se informa en la literatura actual sobre el tema) al martirio, la santidad, los milagros o la evangelización, sino a la formación de una red asociativa entre las poblaciones marginadas, "[...] que cubre un área social totalmente descuidada por la administración romana. Es decir, la lucha organizada por la ciudadanía (avant la lettre, por supuesto) dentro de la sociedad romana" (Hoonaert, 2010: 82).

Con el crecimiento y la consolidación del cristianismo en el imperio romano, la idea de "ciudadanos del reino de Dios" se centró en la educación de los población y los niños, a veces preparando para el bautismo o para el culto en general, como modelo de formación ética y moral, y también en la perspectiva de la legitimación del statu quo establecido por el César ("a César lo que es de César"). Todo esto fue poco a poco impulsando iniciativas en la atención a las demandas de los nuevos cristianos.

Esta lucha organizada proviene de una amplia gama de servicios en el ámbito social y humanitario prestado por los cristianos que en poco tiempo recibió un sólido apoyo popular al mismo tiempo en que se propagaba. En este sentido, los servicios prestados como caridad o bendición, "[...] se traduce, posteriormente, en avances jurídicos, poder político y prestigio cultural" (Hoonaert, 2010: 82). Así, el autor afirma:

\section{[...] El cristianismo no venció por la predicación de los apóstoles y obispos, ni por el destemido testimonio de los mártires, por la santidad de los héroes, por las virtudes o los milagros de sus santos. Ganado, en cambio, por una acción persistente y valiente sobre la base social y política de la sociedad. [...] Consiguió a muchos individuos y grupos una ciudadanía real, aunque limitada y muy modesta acerca de los resultados en términos de la sociedad global (Hoonaert, 2010: 94).}

En la época medieval, las contribuciones en nombre de la sociedad feudal cristiana se vuelven difusas y hay predominio considerable por parte de los señores feudales, lo que, junto al fortalecimiento de la nobleza, deja fuera de cualquier amparo a los siervos y esclavos. En cuanto al Renacimiento, Zeron (2010) destaca que hay una recuperación moderada de la apreciación del hombre como descubridor de sí mismo y del mundo que le rodea. Por lo tanto, se dará énfasis a una educación para la nueva ética cristiana restablecida y en una fase embrionaria, los eventos artísticos y culturales e incluso la estimulación para construir otra perspectiva del mundo mediante el fortalecimiento de la burguesía.

Esto último contribuirá como condicionante para la nueva idea del hombre en formación, de acuerdo a la disposición económica que se produjo en las aguas ultramarinas y que, aun de forma velada, intentaba romper con los intereses de la iglesia tradicional. En este sentido, vale la pena mencionar que la reforma protestante, impulsado por el apoyo de los príncipes alemanes, entre otros, ponen como condición necesaria y esencial para cumplir con las exigencias de la atención a las demandas sociales y la educación para el conocimiento de la palabra de Dios, lo que de alguna manera es una de las iniciativas para pensar en la escuela pública, como ocurrió en la República de Weimar en tiempos posteriores (Lima, 2012). 
Sin embargo, el sentido y el significado de la ciudadanía no correspondía con lo que entendemos hoy en día, incluso más allá de la Edad Moderna (siglo XVII) con la revolución científica, con las transformaciones económicas del siglo XVIII, el fortalecimiento de la burguesía y del iluminismo, durante el siglo XIX con la Revolución Industrial, por un tipo de ciudadano que busca cambiar una tipología de la sociedad existente. Esto se llevará a cabo en su mayor parte a causa de las relaciones de producción y la regulación de la fuerza de trabajo respectivo. Así, poco a poco, en la lucha de clases, el orden estructural de la sociedad que se inaugurara durante la Revolución Industrial, habría que buscar los límites de los derechos y deberes y, por lo tanto, la formación de los significados iniciales de la ciudadanía, en tanto término y categoría social.

No es la sociedad capitalistala que establecerá los parámetros de la ciudadanía, sus sentidos y significados, sino la lucha que hay en su interior, movilizada por las múltiples determinaciones existentes entre medios de producción y fuerza del trabajo.

\section{CIUDADANÍA Y CONTRADICIONES: SENTIDOS Y SIGNIFICADOS}

Para Marshall (1967) la ciudadanía es el hecho de participar de manera integral en la comunidad política, que se manifiesta por la lealtad a la norma actual de la civilización y el acceso al bienestar y la seguridad material ya alcanzados. Sin embargo, la participación en la comunidad política presupone derechos y deberes. Los derechos individuales son equivalentes en la práctica a los indicadores de la ciudadanía en los que el Estado reconoce a la esfera de la producción y el trabajo, la actividad política y el consumo.

Así pues, Marshall cree que la ciudadanía se manifiesta en tres ejes, a saber, derechos civiles, políticos y sociales. Estos elementos interactúan para formar el trípode de la ciudadanía. Cabe explicitar cada elemento.

Los derechos civiles, para Marshall, son los que caracterizan la libertad individual (libertad de pensamiento, las celebraciones de contratos, la adquisición o el mantenimiento de la propiedad): "El elemento civil se compone de los derechos necesarios para la libertad individual - la libertad de movimiento, libertad de prensa, de pensamiento y de fe, el derecho a la propiedad y celebrar contratos válidos y el derecho a la justicia”. Mirado desde esa óptica, en relación al derecho a la justicia, debe haber instrumentos que deben salvaguardar la defensa y mantenimiento de todos los derechos anteriores, por lo que "[...] se diferencia de los demás porque es el derecho de defender y hacer valer los derechos en términos de igualdad con los demás y por el debido encaminamiento procesual" (Marshall, 1967: 63).

Los derechos políticos corresponden a la posibilidad de participación en el poder político (participar de un gobierno, ser electo, elegir el gobierno, votar). Marshall explica: "[...] el derecho a participar en el ejercicio del poder político, como miembro de un órgano investido de autoridad política o como elector de los miembros de ese órgano" (Marshall, 1967: 63). Los derechos sociales se pueden explicitar con la garantía Estatal de un acceso mínimo al bienestar y seguridad material. Los derechos sociales también pueden ser entendidos como el acceso de todas las personas a un nivel de participación en el estándar actual de la civilización:

El elemento social se refiere a todo lo que va desde el derecho a un mínimo de bienestar económico y la seguridad, al derecho a participar plenamente en el patrimonio social y 
llevar la vida de un ser civilizado de acuerdo con las normas vigentes en la sociedad. Las instituciones más íntimas relacionadas con él son el sistema educativo y los servicios sociales (Marshall, 1967: 64).

Cabe señalar que para Marshall los derechos civiles, políticos y sociales sólo se materializan cuando se sobrepasan los textos legales y se efectivizan en el día a día de los individuos sociales. Por lo tanto, el hecho de que esos derechos pueden ser efectuados para hacer cumplir los derechos civiles se hace necesario el acceso a los defensores públicos o los recursos financieros para pagar abogados, la asistencia jurídica a los imposibilitados de costear dicho servicio, la independencia de los magistrados frente a presiones particulares, económica y social. Para el cumplimiento de los derechos políticos a una prerrogativa y la existencia de condiciones de votar y ser votado. Para el cumplimiento de los derechos sociales, el Estado debe tener condiciones financieras suficientes para proporcionar servicios socio-asistenciales para asegurar un mínimo de bienestar social.

Marshall, sin embargo, de manera contundente, afirma de que estos tres elementos que constituyen la garantía de la ciudadanía estaban distanciados en el tiempo por lo que podemos decir que cada uno tiene su génesis en diferentes siglos: los derechos civiles surgen a partir del siglo XVIII, los políticos del siglo XIX y en el siglo XX los sociales. Sin embargo, aclara que dichos períodos deben ser "[...] tratados con una elasticidad razonable, y hay una estrechez, sobre todo entre los dos últimos" (Marshall, 1967: 64).

Para aclarar mejor el proceso evolutivo de los derechos $\mathrm{y}$, paralelamente, de una ciudadanía efectiva, cabe recurrir a las propias palabras de Marshall:

He intentado demostrar que los derechos civiles surgieron en primer lugar y se establecieron de un modo poco similar a la forma moderna que asumieron antes de la entrada en vigor de la primera Ley de Reforma en 1832. Los derechos políticos siguieron a los civiles, y su expansión fue una característica importante del siglo XIX, aunque el principio de ciudadanía política universal no ha sido reconocido sino en 1918. Los derechos sociales, por otro lado, han desaparecido casi por completo en el siglo XVII y principios del XIX. El resurgimiento de estos comenzó con el desarrollo de la educación primaria pública, pero no fue sino hasta el siglo XX que llegó a un pie de igualdad con los otros dos elementos de la ciudadanía (Marshall, 1967: 75).

Sin embargo, Saes (2012), en la búsqueda del significado de la palabra ciudadanía, explica que la evolución de los derechos no es simplemente lineal y simplemente evolutivo, como Marshall propone. La efectuación de estos derechos se produce dentro de la lucha de clases, ya que si las clases trabajadoras, para efectivizar su ciudadanía, asumen una postura dinámica y progresiva, las clases dominantes tienden a ser estagnacionistas y hasta regresivas, haciendo el proceso de creación de los derechos conflictivo, pero no contradictorio. A partir de Göran Terborn, Saes llega a la conclusión de que "[...] serían las luchas populares, potenciadas por las disensiones internas de las clases dominantes nacional e internacional, el factor determinante en el proceso general de creación derechos en la sociedad capitalista".

Saes también se basa en Jean-Claude Delaunay, que comprende el término ciudadanía entre la "ciudadanía efectiva inicial" y la "ciudadanía efectiva evolucionada". La primera se refiere a los derechos civiles necesarios a la implementación, la reproducción y expansión de la relación entre el capital y el trabajo asalariado, firmado como un acuerdo libre de voluntades individuales. El segundo es equivalente a los derechos políticos y sociales. La evolución de la ciudadanía inicial para la ciudadanía efectiva evolucionada 
es el resultado de la capacidad de reivindicación de las luchas populares para imponer a las clases dominantes la efectividad de nuevos derechos. La concesión de tales derechos no destruye al capitalismo, sin embargo, no son deseados por los capitalistas.

De esta manera, en el sentido de comprensión sobre el sentido y el significado social del término, hay que aclarar que la ciudadanía es ante todo un logro de la clase trabajadora y no simplemente una subvención estatal. Así, las luchas sociales aparecen como protagonistas en el proceso de fortalecimiento de los derechos sociales:

Por lo tanto, son las luchas sociales que se llevan a cabo a lo largo de la la Historia y tienen una expresión más fuerte entre los siglos XIX y XX, las que dan lugar a una concepción más amplia de la ciudadanía, significando ahora no sólo los derechos civiles y políticos, sino también los derechos sociales de los trabajadores y trabajadoras (Afonso y Ramos, 2007: 81).

Sin embargo, la efectuación de la ciudadanía o estatus de ciudadano tiene la prerrogativa que une el Estado/nación como regulador de la vida en la sociedad. Cabe aclarar que sólo a partir del final del siglo XVIII el Estado moderno y la Nación moderna se unen para formar el Estado/nación (Afonso y Ramos, 2007). Y, en ese sentido, hay un determinado espacio territorial para la unidad organizacional de un Estado con su propia especificidad: su identidad nacional. Sin embargo, según Afonso y Ramos (2007: 80), "Estado y nación son realidades diferentes, a veces con precedencias, secuencias, vías y protagonismos muy distintos, que pueden o no tejer lazos profundos y duraderos, pero siempre con historicidades propias".

De todos modos, la vinculación del sujeto ciudadano al Estado/nación es prerrogativa para efectivizar la condición de ciudadano, que también es destacado por Benevides:

Ciudadanía y derechos de ciudadanía se refieren a un determinado orden jurídico y político de un país, o de un Estado en el que una Constitución define y garantiza quién es ciudadano, qué derechos y deberes él tendrá, debido a una serie de variables tales como la edad, estado civil, condición de salud física y mental, el hecho de estar o no en deuda con de la justicia penal, etc. Los derechos del ciudadano y la idea misma de ciudadanía no son universales en el sentido de que son fijos a un específico y determinado orden jurídico y político. Por lo tanto, hemos identificado los ciudadanos brasileños, los ciudadanos estadounidenses y los ciudadanos argentinos, y sabemos que varían los derechos y deberes de los ciudadanos de un país a otro (Benevides, 1998: 4).

En este sentido, la autora subraya que la ciudadanía también está vinculada a las decisiones políticas de un gobierno en particular. Así, la ciudadanía puede cambiar de acuerdo a las decisiones políticas, al modificar los derechos y deberes de los ciudadanos, por ejemplo, mediante la modificación del código penal (alterar las sanciones), modificar el código civil (igualar derechos entre los sexos, el cambio en el código de la familia, los derechos y deberes de los cónyuges, hijos, etc.). Todo esto se relaciona con la ciudadanía.

Sin embargo, a lo largo de su proceso histórico, el término "ciudadanía" se ha utilizado con significados diferentes, incluso antagónicos entre sí. Según Martins (2000), la ciudadanía se puede caracterizar en al menos tres manifestaciones: a) en cuanto a valor económico; b) en cuanto a valor gnoseológico y c) en cuanto a valor eminentemente ético y político.

La ciudadanía como valor económico se refiere a la superposición de temas económicos en relación con otros aspectos sociales, haciendo que el ciudadano se reduzca a un mero cliente o un consumidor de los servicios Estatales. 
En cuanto al valor gnoseológico se refiere al ciudadano que simplemente conoce sus derechos y obligaciones, especialmente las relacionadas con el consumo. En este, el individuo adopta una posición de pasividad acrítica, no participa en el proceso de la elaboración de los derechos y deberes ni cuestiona sus principios y su propósito.

En un concepto más amplio que va más allá de la dimensión económica y la gnoseológica, la ciudadanía se presenta con valor ético y político, en el cual el ciudadano está sujeto a su propio destino histórico, se desvincula de la condición del individuo para proyectarse en la lucha política, conoce sus derechos y deberes y participa con una acción consciente y transformadora de la sociedad y el hombre que podría dar lugar a una nueva situación social (Martins, 2000).

En cuanto a la política educativa, señala el mismo autor que, desde las teorías tradicionales hasta las de la contemporaneidad, todas dicen que el objetivo de la educación es la formación de los ciudadanos, pero con características completamente diferentes en cada una de ellas, ya que las políticas sociales son caracterizadas como una injerencia del Estado con el objetivo de mantener las relaciones sociales o el status quo. Así, en una sociedad de clases, el ciudadano se forma de acuerdo a los límites prefijados por los poderes dominantes. Reflejar la relación de la política educativa con la ciudadanía es el objetivo del siguiente acápite.

\section{POLÍTICA EDUCATIVA Y CIUDADANÍA}

El ejercicio de la ciudadanía requiere el conocimiento sobre los derechos y deberes y una postura altamente política de defensa y ampliación de los derechos a través de políticas públicas, de ahí la necesidad de la conciencia colectiva como medio no lineal de "contrato social" que en la actual estructura social, aunque no sea un eje dominante, ha demostrado presencia y fuerza en la dirección de la política educativa desde la perspectiva de la construcción ciudadana con valor ético y político.

Para Afonso y Ramos (2007) la construcción del ciudadano protegido y vinculado a algunos intereses, valores e ideologías particularizados, fue impulsada por la tipología histórica de la escuela pública en Brasil. Estos autores utilizan la nomenclatura ciudadanía restringida a la lógica del Estado-nación para designar, en un momento de la historia humana, las situaciones y las condiciones sociopolíticas en que se caracterizó una ciudadanía no democrática o autoritaria, ya que los derechos legalmente constituidos y respetados fueron escasos o restringidos.

Sin embargo, consideran muy importante el papel de la educación en el proceso de construcción y fortalecimiento de la ciudadanía:

[...] La construcción de la ciudadanía o el proceso de llegar a ser ciudadano y ciudadana es también un proceso histórico y épico que tiene que ver con una victoria contra el poder establecido y que, sin duda, tiene que ver con la educación y la capacidad de tener voz y usar la palabra, y actuar en consecuencia con ella (Afonso y Ramos, 2007: 82).

Sin embargo, en el contexto de la globalización y las reformas educativas en las que los sistemas educativos son delineados a partir de los cambios del sistema capitalista, donde existe una política orientada a satisfacer las necesidades educativas de los sistemas 
de producción, no siempre la ciudadanía como una dimensión de la conciencia colectiva es privilegiada en espacios escolares.

Afonso y Ramos (2007) dicen que es en este contexto que la ciudadanía democrática y la cohesión social emergen contradictoriamente, principalmente porque la ciudadanía se considera un antídoto a la creciente vulnerabilidad neoliberal de los derechos sociales que aumentan la desigualdad y la exclusión social. De ahí la necesidad asumir la escuela como un espacio público de democracia y ciudadanía y como un lugar de confrontación de hegemonías.

Si bien es innegable que la racionalidad económica influyó en la política educativa brasileña, según Sobral (2000) no se puede ignorar la concepción social de la educación, ya que el desarrollo de la política de educación amplía las oportunidades de acceso, que concomitantemente puede disminuir las desigualdades sociales y, por esa razón, se puede afirmar que la educación también promueve la ciudadanía.

Sobral (2000) destaca que la educación promueve el desarrollo de las personas, incluso a través de una mayor competitividad entre ellas, respondiendo a las demandas de las empresas y del país en el mercado internacional. Sin embargo, de este modo, la propia Ley de Directrices y Bases de la Educación, en una lógica dualista, ¿permite desarrollar la educación para la ciudadanía y para la competitividad?

La educación para la ciudadanía parece estar fragilizada. Hay una brecha entre el poder central y las realidades sociales que muestra los límites de los mecanismos formales, verticales, corporativos y clientelista, que se crearon para permitir/impedir la participación de los ciudadanos en los asuntos públicos.

Mirando desde esta óptica, hay una necesidad urgente de que la ciudadanía sea asumida como el punto central para la institucionalización de la participación ciudadana en la toma de decisiones de interés público, ya sea en el contexto de las políticas educativas, así mismo en las demás políticas estructurales e infraestructurales. El desafío principal será fortalecer los espacios deliberativos y modernizar los instrumentos de gestión y articulación.

Por lo tanto, es necesario garantizar el acceso a la información, especialmente a las poblaciones más vulnerables para fortalecer las prácticas de formación de ciudadanía y fomentar una mayor participación en las prácticas de gestión de los asuntos públicos corresponsabilizados, para garantizar la expresión y la representación de los intereses colectivos.

\section{DESAFIOS Y ENCAMINAMIENTOS SOBRE LA DIMENSIÓN DE LA CIUDADANÍA PARA LA EDUCACIÓN EN BRASIL}

Reflexionar sobre los desafíos y encaminamientos de la Política Educativa para una ciudadanía efectiva, sin duda es una tarea compleja, porque son muchos los desafíos que hay que afrontar, como ya fue destacado por varios autores. Recordamos la contribución de Moacir Gadotti (2008), que indica la necesidad de construir una escuela que sea pública, universal $\mathrm{o}$, en otros términos, que sea igual para todos los individuos y que respete la especificidad regional, local y multicultural, que él llama escuela de ciudadanos, cuya elaboración y relaboración deben esforzarse por entender que: 
Para este autor, el principal desafío hoy en día, en lo que respecta a la escuela pública, es proveer un estándar de calidad universal, es decir, igual para todos, y al mismo tiempo respetar las peculiaridades regionales. El mismo aclara que este desafío es el resultado de la nacionalización de la escuela por la burguesía. Expone que para convertirse en escuela ciudadana es necesario "unir lo nacional con lo regional, lo popular con lo público, superando la escuela nacional y estatal para llegar a la escuela popular" (Gadotti, 2008: 52).

Para Gadotti la creación de la escuela ciudadana es un proyecto de creación histórico, un horizonte, una creencia que se puede construir a partir de un decálogo:

1) La escuela pública autónoma es, sobre todo, democrática (para todos) en su gestión, [...] el acceso y la permanencia [...] tiene carácter social comunitario [...]. 2) Para ser autónoma no puede depender de intermediarios, que elaboren políticas y que la tienen una mera ejecutora [...]. 3) La escuela ciudadana debe valorar el contrato de 40 horas con dedicación exclusiva del profesor: 4 horas diarias de clase y 4 de otras actividades y sustituciones (equipo interdisciplinario) [...]. 4) La acción directa. Valorar la iniciativa personal y proyectos de la escuela [...]. 5) La escuela autónoma cultiva la curiosidad, la pasión por el estudio, el gusto por la lectura y producción de textos [...].6) Es una escuela disciplinada [...]. 7) La escuela ya no es un espacio cerrado. Su conexión con el mundo se da con el trabajo [...].

8) La transformación de la escuela no está exenta de conflictos. Ella se da lentamente. [...].

9) No hay dos escuelas iguales. Cada escuela es fruto del desarrollo de sus contradicciones.

10) Cada escuela debe ser lo suficientemente autónoma para poder organizar su trabajo de la manera deseada [...] (Gadotti, 2008: 52-54).

De ese modo, el autor se pregunta qué fuerza podría construir una escuela ciudadana y responde: la transformación de la escuela necesita unir dos movimientos históricos, el movimiento en defensa de la educación pública y el movimiento por una educación popular.

Vaidergorn (2000: 21) advierte que "[...] la educación básica, presentada como un derecho de la ciudadanía conquistada por las masas e incorporada como un valor liberal, no es más que un medio para asegurar la desigualdad social en nombre de la igualdad de derechos. "De ahí la necesidad de conocer el tipo de ciudadanía que supone la Política Educativa. La ciudadanía en el orden social capitalista es un instrumento de estratificación social en la que los liberales lo ven como ciudadanía conservadora, que sirve para los "populista como promesa para el usufructo de los beneficios de la civilización y la posibilidad para superar las diferencias de clase y a los conservadores como elemento de control a las rebeliones de masa, señalando hacia el totalitarismo".

De ahí la importancia de la educación como posibilidad de constitución o formación de una ciudadanía plena. En consecuencia, Valdemarin (2000: 51), basado en los escritos de Rousseau, pretende dar a conocer la interrelación de la política y la educación y señala un camino de acción en el quehacer pedagógico, lo cual pone de relieve la "[...] Necesidad de fortalecimiento individual para evitar dependencia de las reglas y los valores sociales. No al individualismo exacerbado y egoísta practicado hoy en día, sino a la práctica de sus propias potencialidades", para que en el momento de las contradicciones sociales la conciencia colectiva tenga suficiente fundamento y fuerza de contestación y consistencia en sus reclamos.

Esta educación transformadora promueve la conciencia de la mirada de los estudiantes y los profesores, mejora los encaminamientos para que las políticas educativas manifiesten la dimensión democrática inherente a la ciudadanía e incentiva el reconocimiento de todos los participantes de una historia construida a través del tiempo, voz y voto y, más 
que eso, hace que los conocimientos que se adquieren y se despliegan ahora tienen otro sabor: el énfasis en la construcción del hombre como un actor social y no como un sujeto pasivo que simplemente deben consumir un conocimiento intelectual linealizado (Lima, 2010). Este es el gran desafío, entonces: la educación en y para la ciudadanía plena.

Para esta autora, el principio que todavía sirve como una guía para la educación es el que permite el desarrollo de capacidades y la autonomía, en las que los individuos no son capaces de someterse a los poderes establecidos, sin juicio previo.

No someterse a los poderes fácticos, sobre todo si proceden de una sociedad autoritaria, que según Marilena Chaui (Cit. en Nogueira, 2000) es la sociedad brasileña. En esta la ciudadanía es "privilegio de clase", señala la autora.

Nogueira (2000: 108), para discutir el tema de la violencia (otro de los desafíos a enfrentar en la escuela), aclara que la violencia se perpetúa debido a que en la política brasileña sigue habiendo la relación de protección y favor, lo que hace que el espacio público reciba tratamiento privado de los gobernantes, y la escuela ya no sea un lugar donde se construye la ciudadanía, se adquiera derechos, por el contrario, se convierte en un local de promoción personal de los políticos, que se ofrecen como un favor de la clase política al pueblo: "La calidad de esa enseñanza ha sido relegada a la condición de no repitencia y disminución de la evasión, haciendo caso omiso de los aspectos pedagógicos, sus valores, su importancia, sus métodos, sus objetivos y lo más importante la preocupación con la formación del futuro ciudadano".

Como se puso de manifiesto, la educación que propicia la ciudadanía efectiva sigue siendo un gran desafío de la actualidad. Los encaminamientos no sólo se refieren a las políticas públicas que enfatizan la educación como una de las garantías de los derechos de todos los ciudadanos; también se refieren a la experiencia y la comprensión de la ciudadanía como conquista y que, a través de la educación, los hitos son monumentos de la misma, habida cuenta de su dimensión humana, democrática y universal.

\section{CONSIDERACIONES FINALES}

Hemos observado en este estudio que los sentidos y significados de la ciudadanía están plagados de contradicciones. A pesar de que el término ciudadanía existe desde hace mucho tiempo, es debatido desde puntos de vista diferentes. El sentido de ciudadanía que coincide con el punto de vista democrático refuerza los encaminamientos de las políticas educativas para que su efectividad dé sentido a la constitución de ciudadanos que vivan y reclamen el espacio social como conquista universalizada y humanizada, objeto que es el mayor desafío de las sociedades contemporáneas, debido al predominio de la disposición estructural del capitalismo.

Es innegable el reconocimiento de que la educación y la adquisición de conocimientos es fundamental para la construcción de ciudadanía con valor ético y político, pero también es cierto que la escuela por sí sola no es capaz de traducir los cambios sustanciales y estructurales que desee, especialmente en la sociedad brasileña, por otro lado, como dice Lima (2010b), la integración de múltiples voces constituye el tamaño de todo el aprendizaje del hombre, tanto en la escuela de la vida como la vida en la escuela o en el choque de realidades que promueven la desigualdad y la injusticia social. Este proceso, no siendo lineal, demanda de los hombres que viven juntos la redefinición de 
los valores, es decir, a través de la creación de lo que es importante y/o prioritario para el grupo humano o sociedad, van eligiendo los supuestos que guiarán la práctica social, así como la noción de la ética y la moral entre los sujetos. Aquí hay una búsqueda y encaminamiento que las políticas educativas necesitan tratar de forma constante.

\section{REFERENCIAS BIBLIOGRÁFICAS}

Afonso, A. J.; Ramos, E. L.V. (2007). Estado-nación, educación y ciudadana en transición. Revista Portuguesa de Educación, vol.20, n.1, p. 77-98. Web: <http://www.scielo.gpeari.mctes.pt/pdf/ rpe/v20n1/v20n1a04.pdf

Benevides, M.V. (1998). Ciudadanía y Derechos Humanos in Instituto de Estudio Avanzados de la Universidad de São Paulo. Web: <http://www.iea.usp.br/textos/benevidescidadaniaedireitoshumanos.pdf

Funari, P.P. (2010) La ciudadanía entre los romanos In Pinsky,J. y Pinsky, C.B. (orgs.) Historia de la Ciudadanía. 5. ed. Sao Paulo: Contexto.

Gadotti, M. (2008). Escuela Cuidadana. Sao Paulo: Cortez.

Guarinello, N.L. (2010). Ciudad-Estado de la Antigüedad clásica. In Pinsky,J. y Pinsky, C.B. (orgs.), Historia de la Ciudadanía. 5. ed. Sao Paulo: Contexto.

Hoonaert, E. (2010). Las comunidades cristianas de los primeros siglos. In Pinsky,J. y Pinsky, C.B. (orgs.), Historia de la Ciudadanía. 5. ed. Sao Paulo: Contexto.

Lima, P. G. (2010a). Formación de profesores: por una resignificación del trabajo pedagógico en la escuela. Dourados/MS: Editora de UFGD.

Lima, P. G. (2003). Tendencias paradigmáticas en la investigación educativa. Artur Nogueira/ SP: Amilpress.

Lima, P. G. (2010b). Universidad y Educación Básica en el Brasil: la actualidad de Paulo Freire. Dourados/MS: Editora de UFGD.

Lima, P. G. (2012). Fundamentos teóricas y prácticas pedagógicas. Engenheiro Coelho/SP: Imprenta Universitaria Adventista.

Jaeger, W. (1995). Paideia: la formación del hombre griego. Sao Paulo: Martins Fontes.

Marshall, T. H. (1967). Ciudadanía, clase social y estatus. Río de Janeiro: Zahar Editores. Traducción Meton Porto Gadelha.

Martins, M. F.(2000). Una "catarsis" en el concepto de ciudadanía: del ciudadano cliente a la ciudadanía con valor éticos político. Revista de Ética, vol. 2, n. 2, 106-118.

Nogueira, I.S. (2000). La violencia en las escuelas y el desafío de la educación para la ciudadanía. En Vaidergorn, J. (org.), El derecho a tener derechos. Campinas, SP: Autores Asociados

Pinsky, J. (2010). Los profetas sociales y el de Dios de la ciudadanía. En Pinsky ,J. y Pinsky, C.B. (orgs.), Historia de la Ciudadanía. 5. ed. Sao Paulo: Contexto.

Saes, D. A. M. (2012). Ciudadanía y capitalismo: Una aproximación teórica. Sao Pãulo: Instituto de Estudios Avanzados de la Universidad de São Paulo.

Sobral, F.A.F. (2000). Educación para la competitividad o la ciudadanía social?. Perspec., vol.14, n.1, 03-11.

Vaidergorn, J. (2000). Liberalismo, la ciudadanía conservadora y educación. In Vaidergorn, J. (org.), El derecho a tener derechos. Campinas, SP: Autores Asociados.

Valdemarin, V. T.(2000). Educación y política, o la posibilidad de efectuar princ ípios. En Vaidergorn, J. (org.), El derecho a tener derechos. Campinas, SP: Autores Asociados.

Zeron, C.A.(2010). La ciudadanía en Florencia y Salamanca. In Pinsky,J. y Pinsky, C.B. (orgs.), Historia de la Ciudadanía. 5. ed. Sao Paulo: Contexto. 